\title{
Geriatric Anesthesiology: Third Edition
}

\author{
J.G. Reves, Sheila Ryan Barnett, Julie R. McSwain, G. Alec Rooke (Editors). Springer, \\ 2018; 510 pages. ISBN 978-3-319-66877-2
}

\author{
Peter Inglis, BMBS, FRCPC
}

Received: 4 April 2018/Accepted: 5 April 2018/Published online: 18 April 2018

(C) Canadian Anesthesiologists' Society 2018

The third edition of Geriatric Anesthesiology aims to better equip anesthesiologists to deliver comprehensive care for the elderly patient throughout the perioperative period. The ever-expanding role of anesthesiologists in a variety of hospital settings, coupled with a growing population of geriatric patients, makes this topic highly relevant to all anesthesia practitioners. The book is divided into five parts: Fundamentals, System Changes, Pharmacology, Special Concerns, and Postoperative Care. The overall tone of the book emphasizes and encourages the bio-psycho-social model of care that is so vital to directing and delivering proper care to elderly patients.

Part 1 of this third edition, "Fundamentals," lays the foundation for topics that are addressed later in the book. It touches on relevant demographics and theories of aging followed by a detailed discussion of important ethical challenges when caring for the geriatric patient. The issues of decision-making capacity, autonomy, and the goals of care directives are addressed comprehensively.

Part 2, "System Changes," provides an excellent, comprehensive review of the normal physiologic changes associated with aging, and Part 3, "Pharmacology," discusses in detail the application of that information within the framework of the pharmacodynamics and pharmacokinetics of the older patient. All major categories of anesthetic agents are covered. I found the content of these chapters valuable for deepening my understanding of the challenges associated with delivering anesthetics to elderly patients. For example,

P. Inglis, BMBS, FRCPC ( $\square)$

University of Manitoba College of Medicine,

Winnipeg, MB, Canada

e-mail: pjamesinglis@gmail.com often overlooked in anesthesia textbooks, Chapter 14 offers a discussion of how geriatric changes might affect normal wound healing. It also highlights potentially controllable factors - e.g., blood glucose levels, oxygenation, volume status, temperature - that are critically important during the intraoperative and postoperative periods if wound breakdown is to be avoided.

Parts 4 and 5, which cover "Special Concerns" and "Postoperative Care," respectively, address numerous subjects relevant to elderly patients. For example, Chapter 24 offers an excellent review of pacemakers and internal cardiac defibrillators with an emphasis on practical management strategies for patients with these devices. Chapter 31 addresses the highly relevant topic of palliative care from the perspective of an anesthesia provider. It provides a specific discussion and pictorial view of how palliative care can be delivered alongside conventional care. It also addresses the complicated issue of "do not resuscitate" orders during the perioperative period, importantly drawing attention to controversies surrounding various specific interventions, including feeding tubes, noninvasive positive-pressure ventilation, and palliative sedation.

Chapter 30 is dedicated to the extremely important and relevant topics of postoperative delirium and cognitive decline, although these subjects are also touched upon throughout this textbook. Chapter 4 highlights tools with which to predict postoperative delirium and assess the degree of frailty. Chapters 6, 7, and 8 describe strategies such as prehabilitation, "enhanced recovery," and the Hospital Elder Life Program, respectively, which are attractive as potential risk-reducing measures.

Of particular interest to anesthesiologists, Chapter 10 addresses the use of processed electroencephalography and 
the evidence it provides to help prevent postoperative delirium. Chapter 30 offers a comprehensive discussion of the risk factors and pathophysiology of postoperative delirium and cognitive decline. It identifies multiple potentially modifiable risk factors at each stage of the perioperative period. Importantly, the chapter notes that several risk factors can be addressed preoperatively, including poor sleep patterns, malnutrition, untreated pain or depression, ongoing substance abuse, less-than-optimized functional status, polypharmacy, and unwarranted, long, preoperative fasting times. Modifiable intraoperative risk factors include avoidance of excess anesthetic depth and volume administration, benzodiazepines and high-dose opioids, and hypothermia. The key postoperative factors include employing multimodal analgesia, avoidance of deliriogenic medications, and employment of multicomponent prophylactic strategies (e.g., the Hospital Elder Life
Program). The chapter concludes with a short discussion on the treatment of postoperative delirium.

In summary, the Geriatric Anesthesiology: Third Edition is a comprehensive resource for the care of elderly patients from the perspective of an anesthesiologist. It contains clinically relevant information that equips the practitioner with knowledge on how to handle important issues concerning the goals of that care while still offering multiple sections that inform the safe performance of detailed anesthetic strategies with an emphasis on care for the elderly brain. It is undoubtedly a useful resource for all those involved in contemporary anesthetic practice.

Conflicts of interest None declared.

Editorial responsibility This submission was handled by Dr. Hilary P. Grocott, Editor-in-Chief, Canadian Journal of Anesthesia. 